\title{
A New Coarse Grid Correction for RAS/AS
}

\author{
Martin J. Gander ${ }^{1}$, Laurence Halpern², and Kévin Santugini Repiquet ${ }^{3}$
}

\section{Introduction}

It is well known that for elliptic problems, domain decomposition methods need a coarse grid in order to be scalable. One talks about strong scalability of an algorithm, if it permits to solve a problem of fixed size faster in the same proportion that one adds processors. For example if on one processor, a strongly scalable algorithm needs 10 seconds to solve the problem, it would need 1 second using 10 processors. Strong scalability is difficult to achieve already from a theoretical point of view, the limit as the number of processors goes to infinity leads to zero work per processor for a problem of fixed size. One therefore also talks about weak scalability, which means that one can solve a larger and larger problem with more and more processors in a fixed time. For example if a weakly scalable algorithm solves a problem with 100 '000 unknowns in 10 seconds using 1 processor, it should be able to solve a problem with 1'000'000 unknowns in the same 10 seconds using 10 processors. Domain decomposition methods with coarse grids attempt to reach this goal.

The most fundamental result for the two level additive Schwarz method is then precisely that the condition number of the preconditioned elliptic problem satisfies the estimate

$$
\mathscr{K}\left(M_{A S}^{-1} A\right) \leq C\left(1+\frac{H}{\delta}\right),
$$

where $\delta$ denotes the size of the overlap, and $H$ the diameter of the coarse mesh, see the seminal technical report [2], or also the book [11] for a complete and detailed treatment. This result indicates that if one keeps the ratio of the coarse mesh cells to the overlap in a two level additive Schwarz method constant, the method is weakly scalable (as long as the coarse grid solve remains negligible).

Similarly, for substructuring methods, to which the FETI and Balancing NeumannNeumann methods belong, there is a condition number estimate of the preconditioned system of the form

$$
\mathscr{K}\left(M_{\text {sub }}^{-1} A\right) \leq C\left(1+\ln \left(\frac{H}{h}\right)\right)^{2},
$$

where now $h$ denotes the mesh size. This theoretical result has been established for the Balancing Neumann-Neumann algorithm in [3, 9], and for the FETI method in [10]; for a complete treatment, see again the book [11]. In overlapping methods,

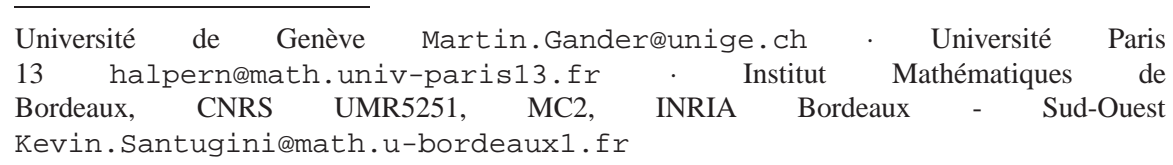




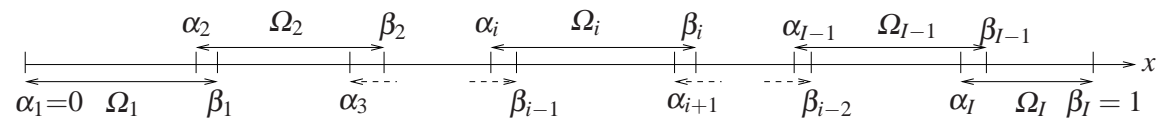

Fig. 1 Decomposition into many subdomains for the one dimensional model problem

the mesh size $h$ is often related to the overlap parameter $\delta$, since the overlap is in general just one or a few mesh cells, and this permits us to compare (1) and (2).

It is also very easily possible to understand intuitively why such a coarse level correction is necessary, if one wants to obtain a scalable method. For the simple model problem,

$$
\left(\eta-\partial_{x x}\right) u=0, \quad u(0) \text { and } u(1) \text { given, }
$$

we consider the parallel Schwarz method introduced by Lions [8] for the decomposition shown in Figure 1,

$$
\begin{gathered}
\left(\eta-\partial_{x x}\right) u_{i}^{n}=0 \quad \text { in } \Omega_{i}, \\
u_{i}^{n}\left(\alpha_{i}\right)=u_{i-1}^{n-1}\left(\alpha_{i}\right), \quad u_{i}^{n}\left(\beta_{i}\right)=u_{i+1}^{n-1}\left(\beta_{i}\right),
\end{gathered}
$$

which is a one level method, and is equivalent to RAS (restricted additive Schwarz [1]), see [4] and [5] for a proof of equivalence. We show in Figure 2 the first few iterations of algorithm (4): in the top row, for the case of two subdomains, we clearly see that both iterates on the left and right subdomain start to converge with the first iterations toward the solution, which is a straight line in this example with $\eta=0$, whereas with sixteen subdomains in the bottom row, the subdomains on the left remain at zero, since communication in this algorithm is only local between the subdomains.

\section{Geometric Investigation of the Coarse Grid Correction}

In order to obtain a scalable algorithm, one can introduce a second level solve like in multigrid: one simply introduces for the fine discretization $A u=f$ of (3) a coarse

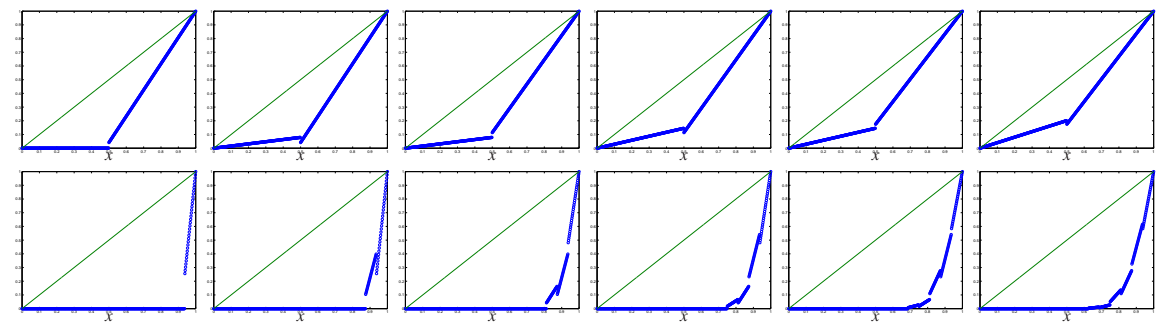

Fig. 2 First iterations of Lions parallel Schwarz methods (equivalent to RAS) for two subdomains in the top row, and sixteen subdomains in the bottom row, $\eta=0$ 


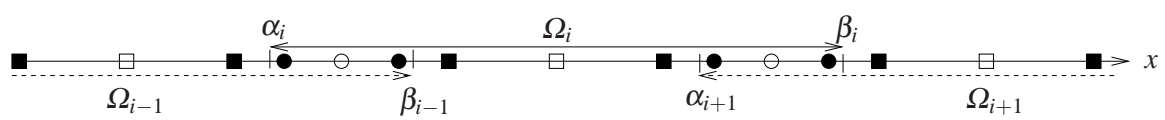

Fig. 3 Various choices to place coarse grid nodes: center of subdomains (empty squares), center of overlaps (empty circles), in the overlap to the left and right of the RAS discontinuity (filled circles) and an equal number of coarse grid points within the subdomains for a fair comparison (filled squares)

grid, and then, after each iteration of algorithm (4), performs the correction

$$
\begin{aligned}
& r_{n}=f-A u_{n} ; \\
& r_{c}=R r_{n} ; \\
& u_{c}=A_{c}^{-1} r_{c} ; \\
& u_{n}=u_{n}+E u_{c} ;
\end{aligned}
$$

using standard components. In our example, we use for the extension $E$ linear interpolation, for the restriction $R$ the extension transposed and normalized, and for the coarse matrix the Galerkin projection $A_{c}=R A E$. A classical choice for the coarse grid is to put one grid point into the center of each subdomain as shown by the empty square in Figure 3. This leads for our example to the convergence result shown in Figure 7 on the left. We clearly see that without coarse grid, the convergence slows down as we add subdomains, whereas with the coarse grid, the convergence curves remain the same, the algorithm is scalable.

In order to see geometrically how the coarse grid correction (5) works, we now visualize in each iteration step how it operates: we show in Figure 4 for the case of four subdomains the iterates before the coarse grid correction, then the residual, the best coarse correction possible and the one actually computed, and finally the iterates after the coarse grid correction. We clearly see that the coarse grid correction is effective: after one coarse grid correction, in the top row, the approximate solution is already very close for all subdomains to the solution sought. We see however also a very unnatural kink appearing in the corrected approximation on the right. Looking at the middle picture of the top row, we see that the residual is concentrated in the center of the overlaps. This is because in RAS, subdomain solutions are composed piecewise, and subdomain solutions satisfy the equations in the subdomains (one says they are harmonic), and thus have zero residual there. The coarse correction computed with grid points in the center of each subdomain are not suitable to correct such a residual support well, as one can see in the middle figure in each row: the residual is smeared out into the subdomains, instead of being corrected in the overlap.

This indicates that coarse grid degrees of freedom in our example should be placed in the overlap, in order to avoid the smearing of the residual into the subdomains, and ideally one should have one degree of freedom on each side of the non-zero residual location, in order to capture the 'jump' in the ideal correction shown in the middle column, see the filled circles in Figure 3. The best coarse space must have as a range such types of corrections. We show in Figure 5 for the same 

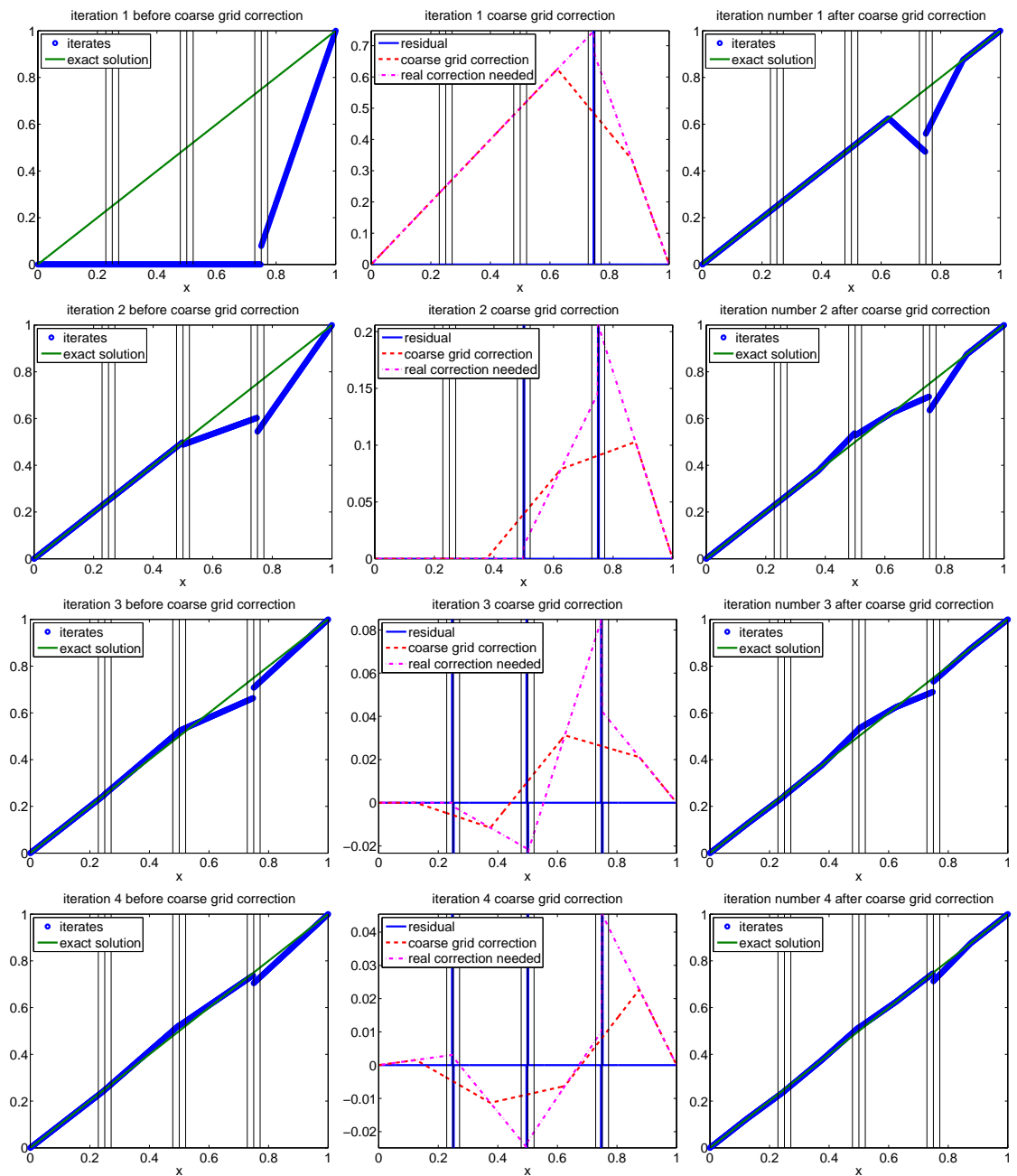

Fig. 4 On each line the iterates before the coarse correction (left), residual, best possible coarse correction and coarse correction actually computed (middle), and iterates after the coarse correction (right) for the first few iterations of the Lions parallel Schwarz method with coarse correction

example what happens with this new coarse grid correction. The result is striking: we obtain convergence of the Schwarz algorithm with this coarse grid correction in two iterations, independently of the number of subdomains. Under the conditions

1. The coarse grid nodes are in the overlap and can capture the discontinuity from RAS,

2. The coarse grid functions satisfy the homogeneous equation,

one obtains a direct solver! In order to illustrate that it is important for the coarse grid shape functions to be harmonic, we show in Figure 6 what happens when we 

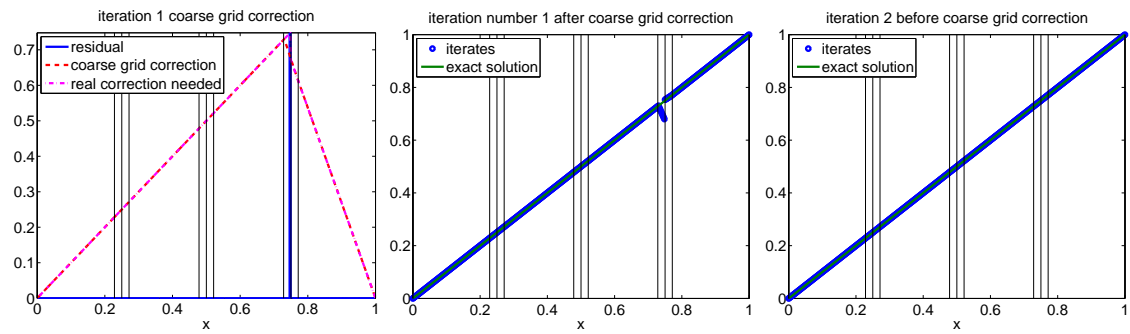

Fig. 5 Residual, best possible coarse grid correction and coarse correction actually computed with good placement of coarse grid nodes (left), iterate after the new coarse grid correction (middle), and iterate after the Schwarz correction (right) starting with the same initial configuration as shown on the top left in Figure 4

solve a problem with $\eta=10$, and still use piecewise linear coarse shape functions. We clearly do not obtain the solution any more after two iterations, but still a very rapidly converging method, note the different scaling in the residual plot on the left of Figure 6! In order to finally compare with a classical two level additive Schwarz method (AS), and measure the influence of using a Krylov method to accelerate the iteration, we present in Figure 7 on the right the convergence histories for this example. It is well known that AS does not converge without Krylov acceleration, which explains the plateau observed in Figure 7. But even with Krylov acceleration, the method is much slower than RAS with the new coarse grid placement. We also notice that RAS now does basically not need Krylov acceleration any more, convergence with and without Krylov is very similar.
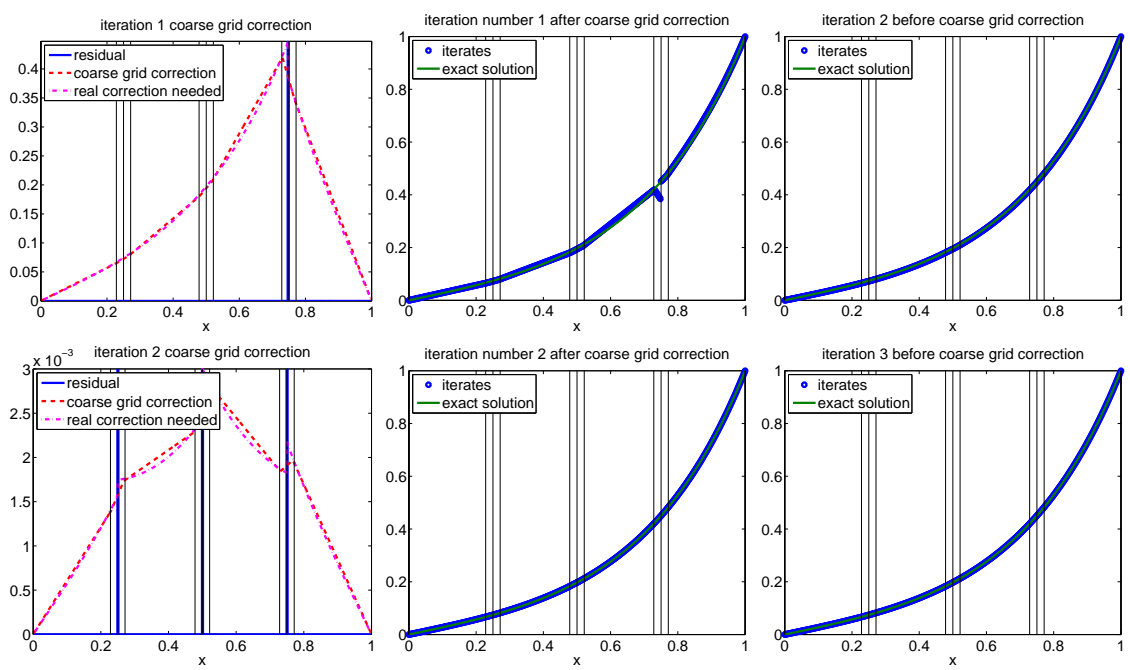

Fig. 6 Example with $\eta=10$, but otherwise the same configuration as in Figure 5 

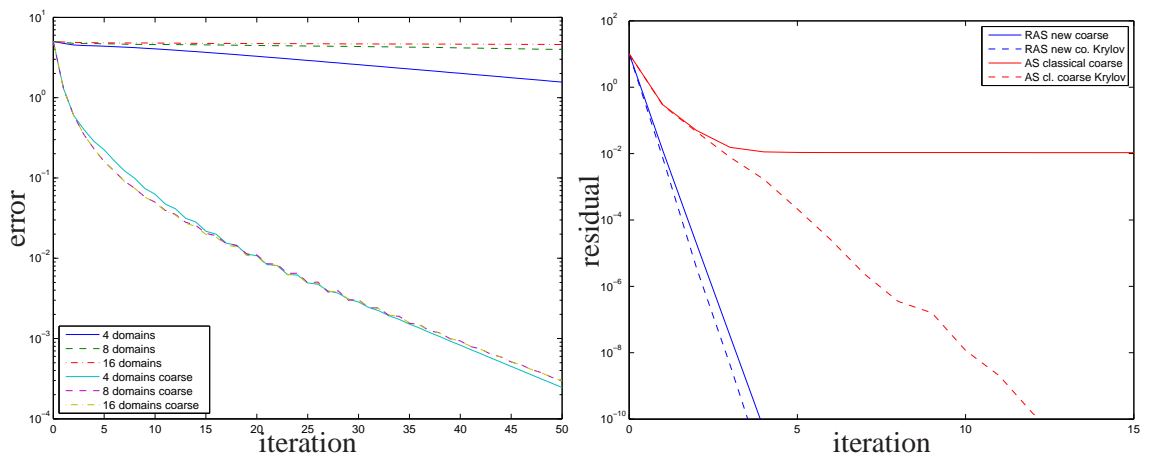

Fig. 7 Iteration versus error for Lions parallel Schwarz algorithm with and without coarse grid on the left, and comparison of AS with classical coarse grid and RAS with optimally placed coarse grid with and without Krylov acceleration on the right

The key question is: can we learn anything from this simple one dimensional example for a problem in higher dimensions? According to the design rule 1. above, the coarse grid needs to have nodes in the overlap, and enough to capture an arbitrary residual located there, as shown in Figure 8 on the left. Then one can prove that we still get a direct solver, provided design rule 2. above is also satisfied. It is interesting at this point to indicate a relation of this coarse grid correction and the optimal transmission operator introduced in [6], which leads to convergence of an optimized Schwarz method in two iterations, independently of the number of subdomains and subdomain configuration, even with crosspoints! The transmission operator also contains a coarse grid component there, and it needs precisely the same traces as our presently proposed coarse grid, and one can find a complete proof at the algebraic level on convergence in two iterations in [6]. Similarly, for a banded matrix, there is also an optimal transmission operator in [7], which again involves
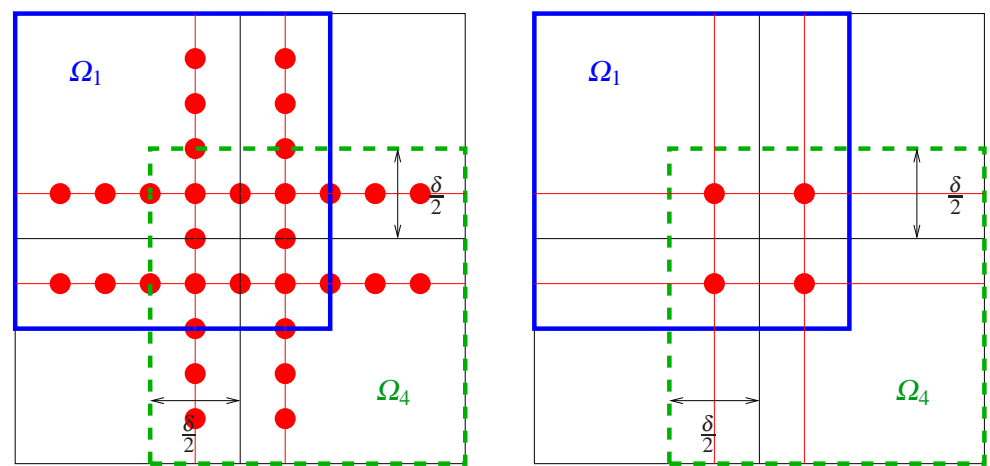

Fig. 8 Optimal coarse grid in two dimensions, and a simple approximation, extending the 1d optimal placement in tensor form 
the same global traces. Naturally, these methods are related, but the precise relation is non-trivial and will be developed elsewhere.

The coarse space indicated in Figure 8 on the left is however very expensive, it requires many degrees of freedom, and also a solve for each in order to obtain harmonic shape functions. A much cheaper alternative is indicated in Figure 8 on the right: one simply places four coarse grid nodes around the cross point of the decomposition. One can then again use Q1 coarse shape element functions, which are harmonic. We show in Figure 9 the convergence histories we obtain for the Laplace equation on the unit square, decomposed into $16 \times 16$ subdomains, using $256 \times 256$ gridpoints. On the left we used the Lions Schwarz method with a coarse grid (equivalent to two level RAS) with overlap $3 h$. We show the result for the

- classical placement of one coarse grid node in the center of each subdomain (classical Q1, empty square in the 1d Figure 3),

- one node at each crosspoint (Q1 in overlap, empty circle in the 1d Figure 3), in order to illustrate that really one node is not enough for the jumps in RAS,

- four nodes per subdomain equally spaced (Q1 fair, filled square in the 1d Figure 3 ) with the same number of coarse grid points as the optimized coarse grid for a fair comparison, and

- four nodes around the crosspoints (optimized Q1, filled circle in the 1d Figure 3), with the same number of coarse grid points as Q1 fair.

Clearly the optimized placement of the coarse grid nodes leads to a substantially faster method than all the other choices.

In Figure 9 on the right we show the corresponding result for AS with minimal overlap $h$. It is interesting to note that for minimal overlap, the influence of the placement of the coarse nodes is even more important, and one obtains a much faster method than with any of the other coarse grid node placements in this two dimensional example.
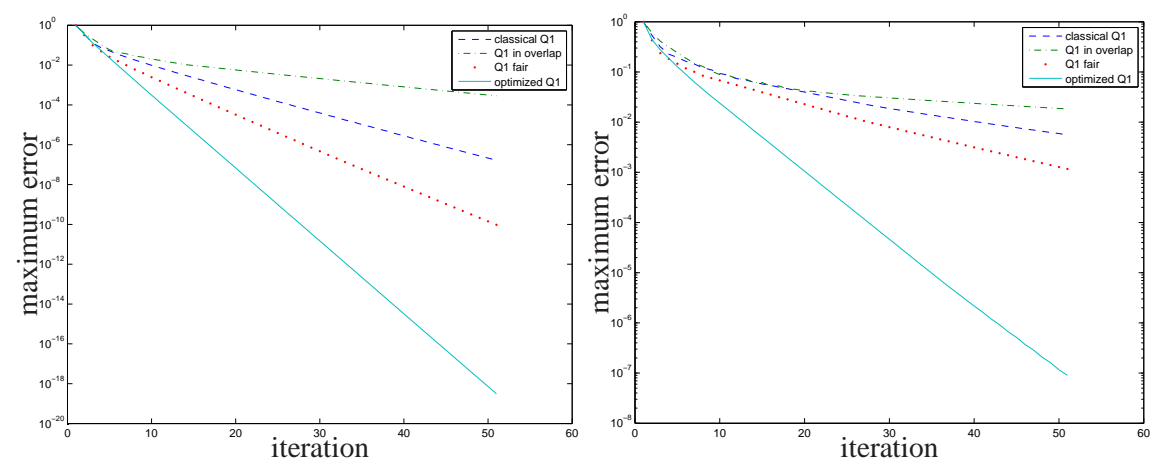

Fig. 9 Convergence histories for two level RAS with various coarse grid node placements on the left and overlap $3 h$, and on the right for AS (additive Schwarz) with overlap $h$ 


\section{Conclusions}

We explained geometrically the interplay between Schwarz iterations and coarse grid corrections. Our example in one dimension revealed that in addition to having harmonic coarse space shape functions, it is also very important where the coarse grid nodes are placed. Optimal placement in one dimension is in the overlap, which leads to a method that converges in two iterations, independently of the number of subdomains. In higher spatial dimensions, it is still possible to construct such a coarse grid correction, but one has to use a number of degrees of freedom proportional to the skeleton of the decomposition. Using however a simple approximation, placing only few degrees of freedom around the crosspoints, leads already to a much faster iterative method than placing coarse nodes as it is done traditionally somewhere within the subdomains. Several theoretical results are already available, though in the different context of transmission conditions, see [6] and [7], and we are currently working on a rigorous error analysis of this new idea. It is also an open question how such an optimized coarse grid would have to look like for a general decomposition of a general domain, our examples here having been simple squares.

\section{References}

1. Xiao-Chuan Cai and Marcus Sarkis. A restricted additive Schwarz preconditioner for general sparse linear systems. SIAM Journal on Scientific Computing, 21:239-247, 1999.

2. Maksymilian Dryja and Olof B. Widlund. An additive variant of the Schwarz alternating method for the case of many subregions. Technical Report 339, also Ultracomputer Note 131, Department of Computer Science, Courant Institute, 1987.

3. Maksymilian Dryja and Olof B. Widlund. Schwarz methods of Neumann-Neumann type for three-dimensional elliptic finite element problems. Comm. Pure Appl. Math., 48(2):121-155, February 1995.

4. Evridiki Efstathiou and Martin J. Gander. Why Restricted Additive Schwarz converges faster than Additive Schwarz. BIT Numerical Mathematics, 43(5):945-959, 2003.

5. Martin J. Gander. Schwarz methods over the course of time. ETNA, 31:228-255, 2008.

6. Martin J. Gander and Felix Kwok. Optimal interface conditions for an arbitrary decomposition into subdomains. In Domain Decomposition Methods in Science and Engineering XIX. Springer LNCSE, 2010.

7. Martin J. Gander, Sebastien Loisel, and Daniel Szyld. An optimal block iterative method and preconditioner for banded matrices with applications to PDEs on irregular domains. SIAM Journal on Matrix Analysis and Applications, 2012. To appear.

8. Pierre-Louis Lions. On the Schwarz alternating method. I. In Roland Glowinski, Gene H. Golub, Gérard A. Meurant, and Jacques Périaux, editors, First International Symposium on Domain Decomposition Methods for Partial Differential Equations, pages 1-42, Philadelphia, PA, 1988. SIAM.

9. Jan Mandel and Marian Brezina. Balancing domain decomposition for problems with large jumps in coefficients. Math. Comp., 65:1387-1401, 1996.

10. Jan Mandel and Radek Tezaur. Convergence of a substructuring method with Lagrange multipliers. Numer. Math., 73:473-487, 1996.

11. Andrea Toselli and Olof Widlund. Domain Decomposition Methods - Algorithms and Theory, volume 34 of Springer Series in Computational Mathematics. Springer, 2005. 